\title{
Peri-membranous ventricular septal defect following His bundle lead implantation
}

\author{
Jan De Pooter ${ }^{1}$, Simon Calle ${ }^{2}$, Thierry Bove ${ }^{1}$, Frédéric Van Heuverswyn ${ }^{3}$, and Frank \\ Timmermans ${ }^{4}$ \\ ${ }^{1}$ University Hospital Ghent \\ ${ }^{2}$ University Hospital Ghent Heart Center \\ ${ }^{3}$ Ghent University Hospital \\ ${ }^{4}$ Univeristy of Ghent
}

April 28, 2020

\begin{abstract}
His bundle pacing (HBP) offers physiologic pacing by placing the pacing lead directly to the His bundle. We present a case in which a HBP lead, implanted at the fragile membranous septum, resulted in a persistent restrictive peri-membranous ventricular septal defect (VSD). This complication of HBP has not been reported before but brings new insights in the discussion regarding the optimal position of a pacing lead in the ventricular septum. The fragility of the membranous septum and low rate of spontaneous closure of membranous VSD, might favor lead placement in the muscular septum when aiming for physiologic pacing.
\end{abstract}

\section{Introduction}

His bundle pacing (HBP) recruits the native His Purkinje system by transmitting the electrical pacing impulse directly to the electrical conduction system of the heart. As such, HBP provides physiologic pacing by avoiding ventricular dyssynchrony as is typically seen with right ventricular (RV) pacing. ${ }^{1-3} \mathrm{HBP}$ requires correct placement of the pacing lead onto the His bundle. The anatomy of the His bundle, its course along the ventricular septum and the amount of adjacent myocardium vary among patients ${ }^{4}$ and may contribute to different electrophysiological pacing responses. Safety issues on HBP mostly considered stability of pacing thresholds and the need for lead revisions. ${ }^{5,6}$ However, there are no reports on how HBP may affect the integrity of the ventricular septum.

\section{Case presentation}

We present the case of a 44-year old male who presented with a syncope and documented symptomatic sinus arrests on an insertable cardiac monitor (Reveal LINQ ${ }^{\mathrm{TM}}$, Medtronic Inc, Minneapolis, MN). Given his young age of the patient and the presence of a first degree atrioventricular block on his twelve lead electrocardiogram, the patient was planned for HBP. HBP was performed by delivering the SelectSecure 3830 pacing lead (Medtronic) through a long preshaped delivery sheat (C315HIS, Medtronic) towards the His region. After localizing a clear His signal with the tip of the pacing lead, the lead was screwed in by 4 to 5 clockwise rotations. At this first HBP implant site (Figure 1, upper panel), HBP resulted in selective HBP (S-HBP) at both high and low output with pacing threshold of $0.5 \mathrm{~V}$ at $1.0 \mathrm{~ms}$ pulse width. This first HBP implant site was accepted as final position. The day after implant, pacing thresholds were stable and the chest X-ray showed correct positioning of the HBP lead after which the patient was dismissed.

At three weeks after implant, the patient presented with exit block on the HBP lead. Interrogation of 
the pacemaker confirmed the absence of HB capture in unipolar pacing configuration and increased pacing thresholds up to $6 \mathrm{~V}$ at $1.0 \mathrm{~ms}$ in bipolar configuration. During lead revision, no macro-dislocation of the HBP lead was observed on fluoroscopy (Figure 1, middle panel) A new HBP lead was guided towards the His bundle region, keeping the first HBP lead in position to serve as anatomical reference (dual lead technique). The first screw attempt of this second HBP lead resulted in non-selective HBP (NS-HBP) at high pacing thresholds and transition towards S-HBP at $1.5 \mathrm{~V}$ at $1.0 \mathrm{~ms}$ (loss of capture at $0.75 \mathrm{~V}$ at $1.0 \mathrm{~ms}$ ). Before slitting the guide sheat of this second HBP-lead, the first HBP lead was removed by 5 counter-clockwise rotations after which the lead came loose and could be completely removed without any resistance. No additional extraction tools were needed. No significant tissue was seen at the tip of the explanted lead. Pacing thresholds for the new HBP lead were stable the day after, but a systolic cardiac murmur was heard upon auscultation. Transthoracic echocardiography revealed the presence of a small perimembranous ventricular septal defect (VSD), located basal to the insertion of the new HBP lead (Figure 1, lower panel). The VSD presented with a restrictive left to right shunt on color Doppler with maximum flow velocitiy of $4,5 \mathrm{~m} / \mathrm{s}$; no aortic regurgitation, right ventricular dilatation or pulmonary hypertension were present. The peri-membranous VSD persisted with stable flow velocities over the left to right shunt during 6 months follow up. As the patient remained asymptomatic without developing hemodynamic sings of pulmonary arterial hypertension, VSD closure was not considered and regular follow-up was proposed. ${ }^{7}$

\section{Discussion}

This case report describes the occurrence of a perimembranous VSD associated with HBP, a complication of HBP which has not been reported previously. HBP becomes widely adopted as an alternative for RV pacing, yielding a clear physiological benefit for pacemaker therapy. Safety assessments of HBP have mainly focused on pacing thresholds, lead dislodgments and the subsequent need for lead revisions. ${ }^{5,}{ }^{6}$ To the best of our knowledge, the impact of HBP leads on the integrity of the interventricular septum has not been investigated. HBP requires the lead to be screwed in at the His bundle region. The His bundle runs along the membranous interventricular septum before dividing into the bundle branches at the crest of the muscular interventricular septum. ${ }^{8}$ However, the course of the His bundle along the membranous septum varies among patients as does the amount of overlying myocardial tissue. Kawashima et al described three distinct types of $\mathrm{HB}$ anatomy and course in relation to the membranous septum. ${ }^{4}$ In type I and type II His bundle anatomy the His bundle is surrounded by a thin and thick layer of myocardial tissue, respectively, and runs on the inferior border of the membranous septum. In patients with a type III His bundle anatomy (25\% of the cases), the His bundle runs just below the endocardial surface of the thin membranous ventricular septum and is not surrounded by myocardial fibers ("naked His bundle"). In those patients, the target zone for HBP, guided by the largest His bundle potential, will guide the implanter towards the thin membranous part of the septum. Placement of a pacing lead at this position might be more prone to lead perforation given the fragility of the membranous. Our case presented with S-HBP at both low and high output at the first HBP implant attempt. As we were not able, even at high pacing output, to capture the septal myocardium (only S-HBP was possible), this patient is suspected to have a type III His bundle anatomy. The absence of macro-dislocation on fluoroscopy and the inability to capture the His bundle on the unipolar tip configuration three weeks after implant, suggests perforation of the HBP lead through the membranous septum. As an echocardiography was not performed before the lead explantation, we cannot exclude that the VSD resulted from the HBP lead removal itself. However, the first HBP lead could be unscrewed easily 3 weeks after implant and was removed with simple traction and without any resistance. Nonetheless, the experience with removal of HBP leads is limited at this moment. Recently, Vijayaraman et al. reported a high success rate of lead extraction of 3830 leads implanted on the HBP. No procedure-related complications occurred in their series, and re-implantation of the HBP lead was successful in the majority of patients, indicating limited damage to the conduction tissue by extraction. However, it was not specified whether the ventricular septum was assessed by echocardiography for occurrence of asymptomatic VSD.

Based on the restrictive character of the VSD in this patient, surgical closure seems not indicated. However, restrictive VSDs might present a future risk for late endocarditis, aortic regurgitation or development of a double-chambered right ventricle and should therefore not be considered harmless. ${ }^{9}$ An iatrogenic 
(peri)membranous VSD is also less likely to close spontaneously. ${ }^{10}$ The incidence and risks of VSD associated with HBP is currently not known, but the occurrence of this complication may trigger the discussion regarding the optimal position of a pacing lead in the ventricular septum to achieve physiological pacing. A HBP lead resulting in NS-HBP, indicating capture of both His Purkinje and adjacent septal myocardial tissue, might be less prone to septal perforation as it probably suggests an implant site more distal towards the thicker muscular septum. Our patient had S-HBP at low and high pacing output, indicating that the lead was presumably screwed in at the thin membranous septum. Of interest, previous studies, comparing S-HBP and NS-HBP, did not report any advantage of S-HBP over NS-HBP in terms of ventricular synchrony or clinical endpoints. ${ }^{11,12}$ Therefore, a better safety profile of NS-HBP with less risk for VSD together with the potential of myocardial capture in case of loss of His bundle capture, might favor HBP implants with non-selective His bundle capture responses. Recently, left bundle branch pacing (LBBP) and left ventricular septal (LVS) pacing have been proposed as an alternative pacing modality to achieve physiologic pacing. ${ }^{13-15}$ With both LBBP and LVSP, the lead is screwed more distally into the muscular septum towards the left side of the septum. Compared to HBP, pacing at the left side of the ventricular septum is associated with lower pacing thresholds, better $\mathrm{R}$ wave sensing, absence of atrial or His bundle oversensing and lead stability. ${ }^{14}$ Although perforations have been reported when targeting a pacing lead towards the left side of the septum, they generally remain without consequences. ${ }^{14}$ Even if a muscular VSD occurs, the chance of spontaneous VSD closure is higher than with membranous VSDs ${ }^{10}$, as closure depends on muscular overgrowth.

Pacing the conduction system or the left side of the septum are novel strategies that should be monitored for rare complications such as VSD. Our case highlights the importance of routine echocardiographic screening for unintended complications that may occur in these novel pacing strategies, especially in the setting of de novo exit block or increased pacing thresholds during follow up.

\section{Conclusion}

This case report describes the occurrence of an iatrogenic peri-membranous VSD as an unexpected complication of HBP. Routine echocardiographic screening of the ventricular septum following pacing lead implantation in the septum is recommended. Insights into the incidence of VSD associated with novel pacing strategies can contribute to the ongoing discussion which pacing site at the septum is best suited for achieving safe and effective physiological pacing.

\section{Figure Legend}

\section{Figure 1:}

Panel A: First His bundle pacing lead (HBP) implant resulting in selective His bundle pacing (S-HBP, bipolar pacing configuration).

Panel B: Lead revision with implantation of a second HBP lead implant using the dual lead technique. The second HBP lead resulted in non-selective HBP (NS-HBP, unipolar configuration), indicating a more distal lead screw in towards the muscular septum.

Panel S: Transthoracic echocardiography showing the peri-membranous ventricular septum defect (yellow asterisk) proximal in the septum and the more distal implanted second HBP lead (yellow arrow). PLAX: parasternal long axis view, PSAX: Parasternal short axis view.

\section{References}

1. Occhetta E, Bortnik M, Magnani A, Francalacci G, Piccinino C, Plebani L, Marino P. Prevention of ventricular desynchronization by permanent para-Hisian pacing after atrioventricular node ablation in chronic atrial fibrillation: a crossover, blinded, randomized study versus apical right ventricular pacing. J Am Coll Cardiol May 16 2006;47:1938-1945.

2. Sharma PS, Dandamudi G, Naperkowski A, Oren JW, Storm RH, Ellenbogen KA, Vijayaraman P. Permanent His-bundle pacing is feasible, safe, and superior to right ventricular pacing in routine clinical 
practice. Heart Rhythm Feb 2015;12:305-312.

3. Abdelrahman M, Subzposh FA, Beer D, Durr B, Naperkowski A, Sun H, Oren JW, Dandamudi G, Vijayaraman P. Clinical Outcomes of His Bundle Pacing Compared to Right Ventricular Pacing. J Am Coll Cardiol May 22 2018;71:2319-2330.

4. Kawashima T, Sasaki H. A macroscopic anatomical investigation of atrioventricular bundle locational variation relative to the membranous part of the ventricular septum in elderly human hearts. Surgical and radiologic anatomy : SRA Aug 2005;27:206-213.

5. Vijayaraman P, Naperkowski A, Subzposh FA, Abdelrahman M, Sharma PS, Oren JW, Dandamudi G, Ellenbogen KA. Permanent His-bundle pacing: Long-term lead performance and clinical outcomes. Heart Rhythm May 2018;15:696-702.

6. Zanon F, Abdelrahman M, Marcantoni L, Naperkowski A, Subzposh FA, Pastore G, Baracca E, Boaretto G, Raffagnato P, Tiribello A, Dandamudi G, Vijayaraman P. Long term performance and safety of His bundle pacing: A multicenter experience. J Cardiovasc Electrophysiol Sep 2019;30:1594-1601.

7. Stout KK, Daniels CJ, Aboulhosn JA, et al. 2018 AHA/ACC Guideline for the Management of Adults With Congenital Heart Disease: A Report of the American College of Cardiology/American Heart Association Task Force on Clinical Practice Guidelines. J Am Coll Cardiol Apr 2 2019;73:e81-e192.

8. Anderson RH, Yanni J, Boyett MR, Chandler NJ, Dobrzynski H. The anatomy of the cardiac conduction system. Clinical anatomy (New York, NY) Jan 2009;22:99-113.

9. Neumayer U, Stone S, Somerville J. Small ventricular septal defects in adults. Eur Heart J Oct 1998;19:1573-1582.

10. Turner SW, Hunter S, Wyllie JP. The natural history of ventricular septal defects. Arch Dis Child Nov 1999;81:413-416.

11. Zhang J, Guo J, Hou X, Wang Y, Qian Z, Li K, Ge P, Zou J. Comparison of the effects of selective and non-selective His bundle pacing on cardiac electrical and mechanical synchrony. Europace Jun 1 2018;20:1010-1017.

12. Beer D, Sharma PS, Subzposh FA, et al. Clinical Outcomes of Selective Versus Nonselective His Bundle Pacing. JACC Clin Electrophysiol Jul 2019;5:766-774.

13. Li Y, Chen K, Dai Y, Li C, Sun Q, Chen R, Gold MR, Zhang S. Left bundle branch pacing for symptomatic bradycardia: Implant success rate, safety, and pacing characteristics. Heart Rhythm Dec 2019;16:1758-1765.

14. Vijayaraman P, Subzposh FA, Naperkowski A, Panikkath R, John K, Mascarenhas V, Bauch TD, Huang W. Prospective evaluation of feasibility, electrophysiologic and echocardiographic characteristics of left bundle branch area pacing. Heart Rhythm May 252019.

15. Mafi-Rad M, Luermans JG, Blaauw Y, Janssen M, Crijns HJ, Prinzen FW, Vernooy K. Feasibility and Acute Hemodynamic Effect of Left Ventricular Septal Pacing by Transvenous Approach Through the Interventricular Septum. Circulation Arrhythmia and electrophysiology Mar 2016;9:e003344. 


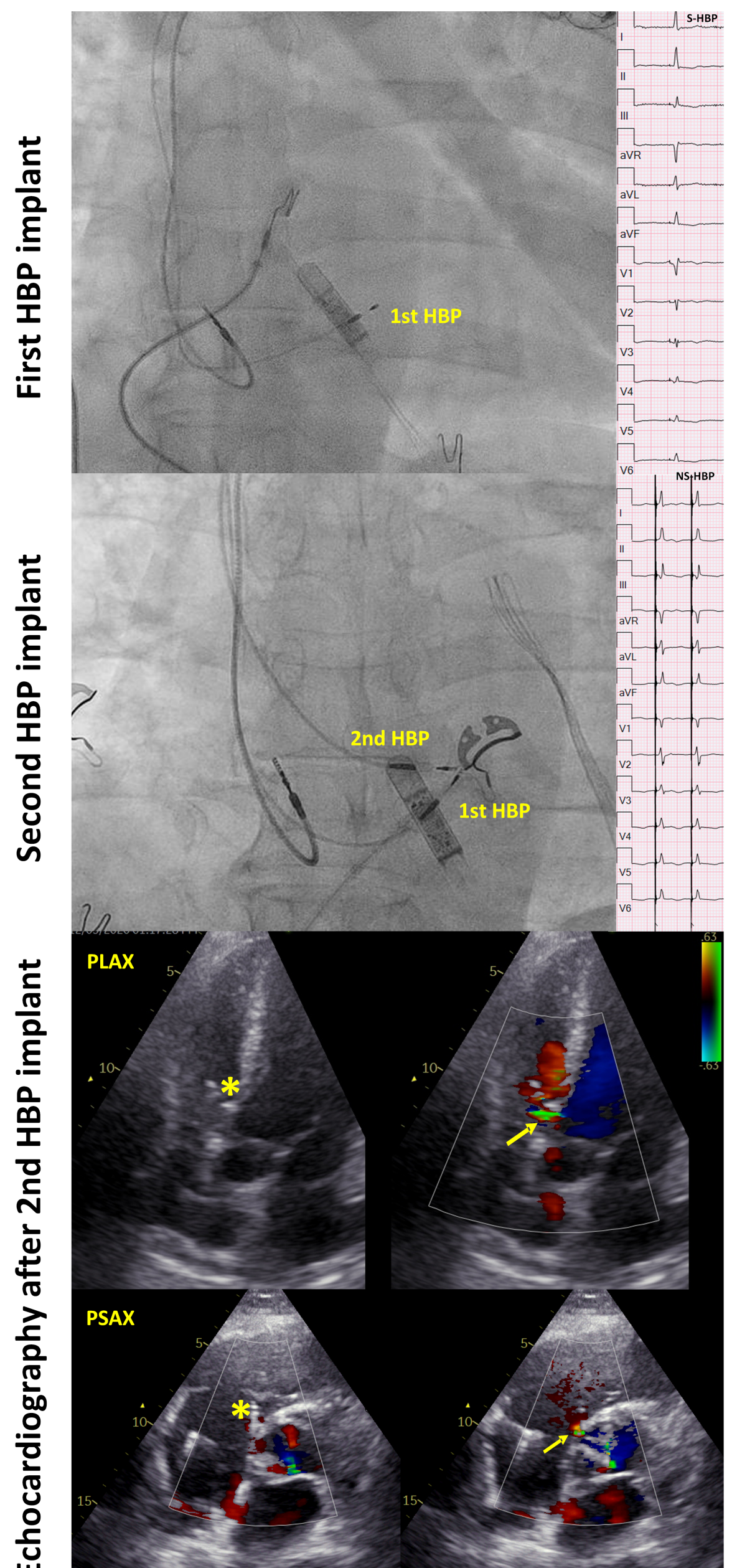

